\title{
Niveles de competencias en auditoría de servicios de salud de los médicos del primer nivel de atención del distrito de Comas, 2018
}

Erick Nelson Guerra Uriarte* 1,2,a

\section{RESUMEN}

Objetivo: Identificar el nivel de competencias en auditoría de servicios de salud de los médicos del primer nivel de atención del distrito de Comas en 2018.

Materiales y métodos: Estudio de tipo analítico. De una población total de 110 médicos, intervinieron 32 individuos quienes accedieron a participar en el estudio. Se evaluaron las variables sociodemográficas y los cinco tipos de competencias en auditoria de servicios de salud: las cuatro primeras fueron graduadas como "Aprobado", "En proceso" y "Desaprobado", mientras que la quinta fue calificada en los niveles "Básico”, "Intermedio" y "Avanzado”. Los datos se procesaron con el software SPSS versión 24.00.

Resultados: De la población evaluada, el 62,50 \% fueron médicos asistenciales y 37,50 \%, jefes de establecimientos. Predominaron los hombres $(68,75 \%)$ respecto a mujeres $(31,25 \%)$. El resultado global del nivel de competencia de auditoría de servicios de salud fue “Desaprobado” (50,00 \%), “En proceso” (43,75 \%) y “Aprobado” (6,25 \%).

Conclusiones: El 6,25 \% de las personas evaluadas alcanzaron el nivel "Aprobado" en las competencias de auditoría de servicios de salud. Las variables "Años laborando" (Sig. = 0,011) y "Formación general” $($ Sig. = 0,031) tienen relación significativa con el desarrollo de las competencias en auditoría de servicios de salud.

Palabras clave: Competencia profesional; Primer nivel de atención; Auditoría administrativa (Fuente: DeCS BIREME).

\section{Levels of health services audit proficiency among primary health care physicians from Comas district, 2018}

\section{ABSTRACT}

Objective: To identify the levels of health services audit proficiency among primary health care physicians from Comas district in 2018.

Materials and methods: An analytical study was conducted. Out of a population of 110 physicians, 32 accepted to participate in the study. Sociodemographic variables and five types of health services audit proficiency were assessed: the first four were graded as "pass," "in process" and "fail," while the fifth was scored as "basic," "intermediate" and "advanced." The data was processed using SPSS Statistics statistical software version 24.00.

Results: Sixty-two point five zero percent $(62.50 \%)$ of the study population consisted of attending physicians, while $37.50 \%$ were medical chief officers. Males (68.80\%) prevailed over females $(31.30 \%)$. The global result of the levels of health services audit proficiency was “fail” (50.00\%), “in process” (43.80\%) and “pass” (6.25\%).

Conclusions: Six point two five percent (6.25\%) of the study population achieved the "pass" score in the health services audit proficiency. The variables "tenure" (Sig. =0.011) and "general training" (Sig. =0.031) were significantly related to the development of health services audit proficiency.

Keywords: Professional competence; Primary health care; Management audit (Source: MeSH NLM).

1 Dirección de Redes Integrales de Salud Lima Norte-DIRIS LN. Lima, Perú.

2 Red de Salud Comas. Lima, Perú.

a Médico cirujano, auditor.

*Autor corresponsal. 


\section{INTRODUCCIÓN}

Es de conocimiento general que la labor de dirección que ejercen los titulares de las instituciones prestadoras de servicios de salud (IPRESS) públicas busca la sostenibilidad, crecimiento y cumplimiento de metas institucionales. No es una labor sencilla, toda vez que las dificultades propias del sector y las exigencias particulares de este nivel de atención afectarían el desempeño de dichos profesionales, por lo que, para ejercer adecuadamente un puesto de dirección a este nivel, es necesario contar con una serie de competencias en gestión y control que garanticen un correcto desempeño, ya que, como menciona Mona Shah (1), "las competencias son habilidades únicas relacionadas con un desempeño laboral superior".

Asimismo, es importante mencionar que en los últimos años la auditoría en el sector salud ha tomado mayor importancia como instrumento de control y se ha convertido en una gran aliada de la mejora continua de los procesos relacionados a la práctica médica y salud pública ${ }^{(2,3)}$.

Por otro lado, la calidad de los servicios de salud también ha tomado una gran importancia en el país, tanto es así que en la actualidad el Ministerio de Salud (Minsa), mediante el documento técnico "Sistema de Gestión de la Calidad en Salud" (4), aprobado con Resolución Ministerial N 519-2006/ MINSA de 30 de mayo del 2006, tiene como objetivo general mejorar continuamente la calidad de los servicios, recursos y tecnología del sector salud, por lo que estructuró dicho sistema en los cuatro componentes siguientes:

- Planificación para la calidad.

- Organización para la calidad.

- Información para la calidad.

- Garantía y mejora.

En ese sentido, el presente estudio se basó en el elemento "Proceso de acreditación de la institución prestadora de servicios de salud-IPRESS", del componente "Garantía y mejora", ya que permite al evaluador tener una visión general respecto a la dirección, funcionamiento, control y calidad de los servicios que brindan las IPRESS debido a que este proceso consiste en la evaluación de comparación del desempeño de la IPRESS con estándares conocidos, óptimos y factibles de alcanzar ${ }^{(4)}$.

Este artículo se enmarca en el contenido de la tesis Las competencias en auditoria de los médicos generales y el vínculo con la calidad del proceso gerencial de las instituciones prestadoras de servicios de salud del primer nivel de atención de la dirección de redes integradas Lima Norte-2018 para optar por el grado de Magíster en la maestría Gerencia en los Servicios de Salud de la Universidad Nacional Mayor de San Marcos.

\section{MATERIALES Y MÉTODOS}

\section{Diseño y población}

Estudio de tipo no experimental, cuantitativo, analítico y de corte transversal. La población estudiada fueron los médicos generales del primer nivel de atención del distrito de Comas. Se incluyó a treinta y dos participantes de un universo de 110 médicos. Los profesionales que decidieron no completar el instrumento de recolección de datos y los que no estuvieron de turno durante la visita al establecimiento fueron excluidos. La información se ingresó a la base de datos SPSS versión 24 .

\section{Variables y mediciones}

Los participantes completaron el instrumento sobre competencias en auditoría de servicios de salud, que fue elaborado por el autor de acuerdo a los productos obtenidos de los procesos evaluados durante la acreditación de los establecimientos del primer nivel de atención. Estos productos fueron estandarizados y evaluados mediante el "Listado de estándares de acreditación para establecimientos de salud y servicios médicos de apoyo de la norma técnica N ${ }^{\circ}$ 050-MINSA/DGSP-V02" (5), aprobada con Resolución Ministerial N456.2007/MINSA, el 4 de junio del 2007.

Para la investigación, el instrumento fue sometido a juicio de expertos para determinar la validez de contenido, de constructo y de criterio. Luego se realizó una prueba piloto para determinar si el instrumento era amigable para los participantes.

El instrumento fue dividido en dos partes, la primera recolectó información respecto a características sociodemográficas:

-Sexo
-Modalidad laboral
- Jefatura
-Años laborales
- Formación en auditoría
- Formación gerencial

La segunda parte recolectó datos específicos relacionados a las siete competencias básicas en auditoría de servicios de salud descritas por Mónica S. Chirinos-Muñoz ${ }^{(6)}$ :

- Dominio de normativas y aspectos legales que rigen el sector salud y los procesos.

-Dominio de conocimientos en métodos de trabajo y técnicas de estudio, redacción y síntesis.

- Dominio de programas de informática y de computación en general.

-Dominio de tareas y procesos profesionales asistenciales, administrativos (general y especializado).

- Manejo y destreza en la aplicación de metodología 
Niveles de competencias en auditoría de servicios de salud de los médicos del primer nivel de atención del distrito de Comas, 2018

científica en salud que apoye el análisis y la consistencia en la información.

- Dominio del o los idiomas que sean parte de la dinámica de trabajo de la organización.

-Dominio de técnicas estadísticas.

Para los fines del presente estudio, las siete competencias fueron adecuadas a cinco, toda vez que tres de ellas están relacionadas al desarrollo de competencias analíticas y de segunda lengua, como las considera Araya-Pizarro (7), por lo que se decidió agruparlas en una sola, y quedó a denominación siguiente:

- Dominio de normativas y aspectos legales que rigen el sector salud y los procesos. (i1)

- Dominio de conocimientos en métodos de trabajo y técnicas de estudio, redacción y síntesis. (i2)

-Dominio de tareas y procesos profesionales asistenciales, administrativos (general y especializado). (i3)

- Manejo y destreza en la aplicación de metodología científica en salud que apoye el análisis y la consistencia en la información. (i4)

- Dominio de técnicas estadísticas, programas de informática y de computación en general e idiomas que sean parte de la dinámica de trabajo de la organización. (i5)

Las competencias se evaluaron según los criterios de la "Guía técnica para la identificación de competencias y diseño de estándares e instrumentos de evaluación de competencias específicas con el enfoque funcional" (8). Para evaluar los procesos se utilizó la "Guía técnica del evaluador para la acreditación de establecimientos de salud y servicios médicos de apoyo" (9), la cual clasifica los macroprocesos de la siguiente manera:

- Direccionamiento

- Gestión recursos humanos

- Gestión de la calidad

- Manejo de riesgo de la atención

- Gestión de seguridad ante desastres

- Control de la gestión y prestación

Asimismo, las primeras cuatro competencias fueron graduadas de la manera siguiente:

-Desaprobado : $<60 \%$

- En proceso : $<60 \%-85 \%$

-Aprobado : > $85 \%$
Además, la competencia i5 fue calificada en los niveles siguientes:

\section{-Básico \\ - Intermedio \\ -Avanzado}

\section{Análisis estadístico}

Los datos obtenidos fueron codificados, registrados en una base de datos y procesados con el paquete estadístico SPSS 24. Se aplicó la prueba de exacta de Fisher para determinar si había asociación entre las variables.

\section{Consideraciones éticas}

La información obtenida a través del instrumento durante la investigación se mantuvo bajo un estricto nivel de seguridad y confidencialidad.

\section{RESULTADOS}

Los resultados obtenidos de la aplicación del instrumento a los profesionales médicos nos permiten presentar los siguientes resultados.

\section{Caracterización de la población objeto de estudio}

En la tabla 1 se muestra que el $68,75 \%$ de los participantes fueron varones y el $31,25 \%$, mujeres. Asimismo, hallamos que el $43,75 \%$ pertenecen al régimen CAS; el $34,38 \%$, al régimen 276 , y el $21,88 \%$ son locadores de servicios. La experiencia laboral es menor de 3 años en el $46,88 \%$ de participantes, y mayor de 3 en el $53,13 \%$.

Por otro lado, se observó que algunos participantes poseían formación en auditoría en servicios de salud en la modalidad de diplomado $(37,50 \%)$, y en maestría y doctorado $(3,13 \%)$, mientras que el 59,38\% manifestó no tener formación en dicha área. Mientras que en el campo de gerencia de servicios de salud, el $40,63 \%$ de los participantes realizó un diplomado, el 9,38 \% ha cursado una maestría o diplomado y el 50,00 \% no tuvo formación en esta área.

Al momento de la aplicación del instrumento, el 37,50 \% de participantes tuvo el cargo de jefe de establecimiento, mientras que el $62,50 \%$ solo eran médicos asistenciales. 
Tabla 1. Características sociodemográficas de los médicos

\begin{tabular}{|c|c|c|c|}
\hline & \multicolumn{2}{|l|}{ Frecuencia } & $\begin{array}{c}\text { Porcentaje } \\
(\%)\end{array}$ \\
\hline \multicolumn{4}{|l|}{ Jefe } \\
\hline & Sí & 12 & 37,50 \\
\hline & No & 20 & 62,50 \\
\hline \multicolumn{4}{|l|}{ Sexo } \\
\hline & Masculino & 22 & 68,75 \\
\hline & Femenino & 10 & 31,25 \\
\hline \multicolumn{4}{|l|}{ Modalidad laboral } \\
\hline & CAS & 14 & 43,75 \\
\hline & Nombrado & 11 & 34,38 \\
\hline & N/A & 7 & 21,88 \\
\hline \multicolumn{4}{|l|}{ Años laborales } \\
\hline & $<3$ & 15 & 46,88 \\
\hline & $>3$ & 17 & 53,13 \\
\hline \multicolumn{4}{|l|}{ Formación auditora } \\
\hline & Diplomado & 12 & 37,50 \\
\hline & Maestría/doctorado & 1 & 3,13 \\
\hline & $\mathrm{N} / \mathrm{A}$ & 19 & 59,38 \\
\hline \multicolumn{4}{|l|}{ Formación gerencial } \\
\hline & Diplomado & 13 & 40,63 \\
\hline & Maestría/doctorado & 3 & 9,38 \\
\hline & $\mathrm{N} / \mathrm{A}$ & 16 & 50,00 \\
\hline
\end{tabular}

\section{Nivel de competencias}

En relación al desarrollo del nivel global de las competencias en auditoria de servicios de salud, se evidenció que el 50,00\% de los participantes se ubicó en la categoría "Desaprobado"; el 43,80 \%, “En proceso”, y el 6,30\%, en “Aprobado”. Estos resultados advierten que la mayoría de médicos participantes no tendrían las competencias necesarias para desempeñarse de manera adecuada, en el caso eventual de que tuvieran que asumir funciones de control de la calidad de los servicios de salud e, inclusive, la dirección de un establecimiento de salud del primer nivel de atención (Figura 1 y tabla 2).

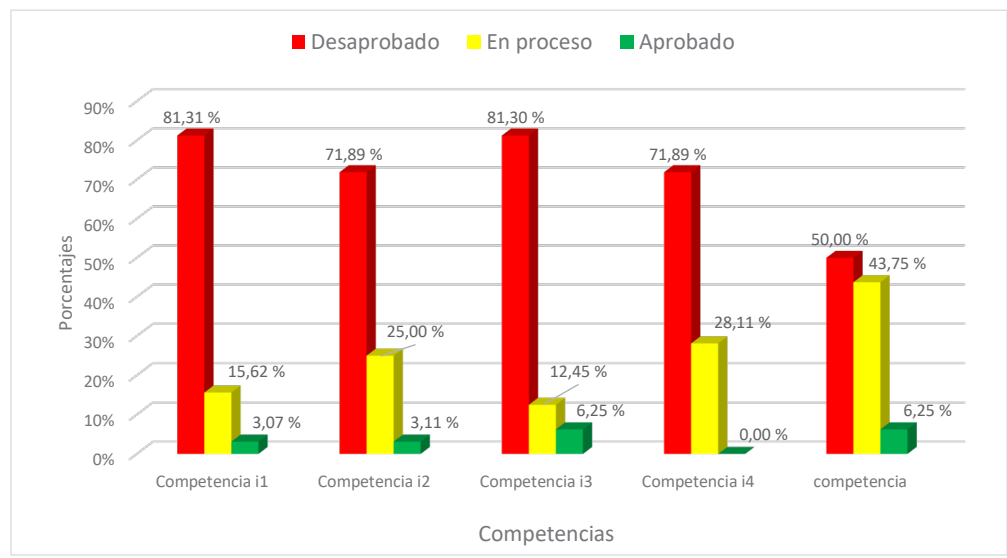

Figura 1. Características de los médicos según su nivel de competencia $(n=32)$ 
Niveles de competencias en auditoría de servicios de salud de los médicos del primer nivel de atención del distrito de Comas, 2018

Tabla 2. Características de los médicos según su nivel de competencia

\begin{tabular}{|c|c|c|c|c|c|c|c|c|c|c|}
\hline & \multicolumn{2}{|c|}{ Competencia i1 } & \multicolumn{2}{|c|}{ Competencia i2 } & \multicolumn{2}{|c|}{ Competencia i3 } & \multicolumn{2}{|c|}{ Competencia i4 } & \multicolumn{2}{|c|}{ competencia } \\
\hline & $n$ & $\%$ & $\mathrm{n}$ & $\%$ & $n$ & $\%$ & $n$ & $\%$ & $n$ & $\%$ \\
\hline Desaprobado & 26 & $81,31 \%$ & 23 & $71,89 \%$ & 26 & $81,30 \%$ & 23 & $71,89 \%$ & 16 & $50,00 \%$ \\
\hline En proceso & 5 & $15,62 \%$ & 8 & $25,00 \%$ & 4 & $12,45 \%$ & 9 & $28,11 \%$ & 14 & $43,75 \%$ \\
\hline \multirow[t]{2}{*}{ Aprobado } & 1 & $3,07 \%$ & 1 & $3,11 \%$ & 2 & $6,25 \%$ & 0 & $0,00 \%$ & 2 & $6,25 \%$ \\
\hline & 32 & $100,00 \%$ & 32 & $100,00 \%$ & 32 & $100,00 \%$ & 32 & $100,00 \%$ & 32 & $100,00 \%$ \\
\hline
\end{tabular}

En la tabla 2 se observa que, de la competencia i1, el $81,31 \%$ de los participantes está "Desaprobado", el 15,62 \% se encuentra “En proceso" y el 3,07 \% está "Aprobado". Estos resultados llaman la atención debido a que esta competencia se relaciona al conocimiento del ordenamiento jurídico que rige el funcionamiento de los establecimientos del sector salud.

Respecto a la competencia i2, relacionada al conocimiento de la normativa de recursos humanos, el 71,89\% de los participantes se encuentra "Desaprobado"; el 25,00 \%, “En proceso"; y el 3,11 \% está “Aprobado". Este hallazgo denotaría una brecha de conocimiento en relación a la gestión del capital humano.

Así también, se pudo observar que en los resultados de la competencia i3 el 6,25\% de los participantes están “Aprobados"; el 12,45 \%, “En proceso”, y “Desaprobado", el 81,30\%; estas cifras también son preocupantes porque esta competencia se relaciona al ordenamiento jurídico que regula las políticas, estrategias y programas de salud asistenciales del primer nivel de atención.

La competencia i4, referida a los métodos de investigación y análisis, muestra que el 71,89\% de los participantes desaprobó, el 28,11 \% se clasificó en el nivel “En proceso" y ningún participante aprobó. Estos resultados son llamativos debido a que el primer nivel de atención tiene como función la prevención de enfermedades, y debe brindar información confiable a los sistemas de vigilancia del Minsa.

Por otro lado, para la competencia i5 (relacionada al dominio de estadística, herramientas informáticas y conocimiento de otras lenguas) se observó que el 90,62\% de los participantes alcanzaron el nivel básico; el 9,38\%, el nivel intermedio; mientras que ningún médico alcanzó el nivel avanzado (Tabla 3 y figura 2).

Tabla 3. Características de competencias i5

\begin{tabular}{|lrr|}
\hline & \multicolumn{2}{c}{ Competencia i5 } \\
Niveles & $\mathrm{n}$ & \multicolumn{1}{c|}{$\%$} \\
\hline Básico & 29 & 90,62 \\
\hline Intermedio & 3 & 9,38 \\
\hline Avanzado & 0 & 0,00 \\
\hline & 32 & 100,00 \\
\hline
\end{tabular}

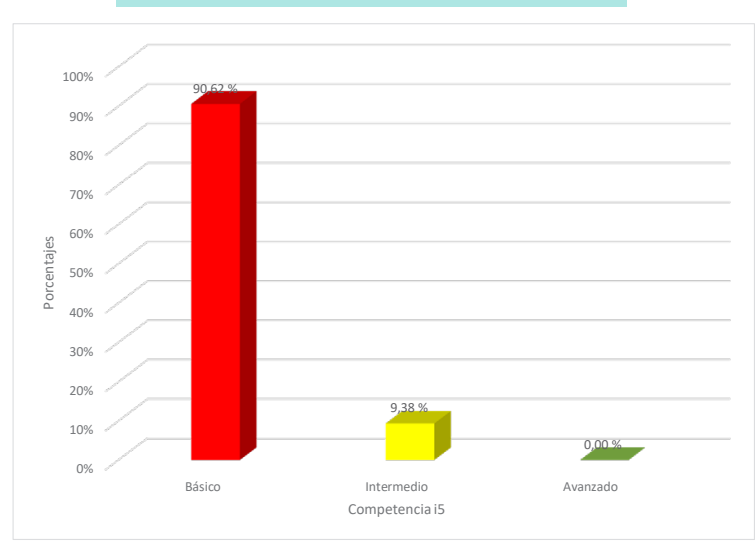

Figura 2. Características de competencia i5 $(n=32)$ 
Al aplicar la prueba exacta de Fisher se encontraron valores de Sig. $=0,011(<0,05)$ para la variable "Años laborando", y Sig. $=0,031(<0,05)$ para la variable "Formación general" (formación en auditoría y gerencial). Estos resultados determinarían que existe una relación significativa entre dichas variables y el nivel de competencia en auditoría de los servicios de salud (Tabla 4).

Tabla 4. Variables sociodemográficas de los médicos del primer nivel de atención

\begin{tabular}{|c|c|c|c|c|c|c|c|c|c|c|}
\hline & & & & el de & ompete & & & & & Prueba \\
\hline & & & roceso & & medio & & etente & & Total & \\
\hline & & $n$ & $\%$ & $\mathrm{n}$ & $\%$ & $\mathrm{n}$ & $\%$ & $\mathrm{n}$ & $\%$ & \\
\hline Sexo & Masculino & 11 & 50,00 & 10 & 45,45 & 1 & 4,55 & 22 & 100,00 & $\mathrm{Sig}=1,000$ \\
\hline & Femenino & 5 & 50,00 & 4 & 40,00 & 1 & 10,00 & 10 & 100,00 & \\
\hline Modalidad laboral & CAS & 7 & 50,00 & 5 & 35,71 & 2 & 14,29 & 14 & 100,00 & Sig $=0,373$ \\
\hline & Nombrado & 4 & 36,36 & 7 & 63,64 & 0 & 0,00 & 11 & 100,00 & \\
\hline & N/A & 5 & 71,43 & 2 & 28,57 & 0 & 0,00 & 7 & 100,00 & \\
\hline Años laborando & Menos de 3 años & 10 & 66,67 & 3 & 20,00 & 2 & 13,33 & 15 & 100,00 & $\operatorname{Sig}=0,011$ \\
\hline & 3 años a más & 6 & 35,29 & 11 & 64,71 & 0 & 0,00 & 17 & 100,00 & \\
\hline Jefe & Sí & 6 & 50,00 & 5 & 41,67 & 1 & 8,33 & 12 & 100,00 & $\operatorname{Sig}=1,000$ \\
\hline & No & 10 & 50,00 & 9 & 45,00 & 1 & 5,00 & 20 & 100,00 & \\
\hline Formación general & Diplomado & 4 & 30,77 & 9 & 69,23 & 0 & 0,00 & 13 & 100,00 & $\operatorname{Sig}=0,031$ \\
\hline & Maestría/doctorado & 1 & 33,33 & 1 & 33,33 & 1 & 33,33 & 3 & 100,00 & \\
\hline & N/A & 11 & 68,75 & 4 & 25,00 & 1 & 6,25 & 16 & 100,00 & \\
\hline
\end{tabular}

\section{DISCUSIÓN}

De los resultados se observó que la población con experiencia laboral mayor a 3 años tuvo una ligera diferencia a favor frente a la que tuvo menos de 3 años. Estos son similares a los encontrados por Claus Michael Preger (10), quien determinó que los médicos auditores del estado de Río Grande en Brasil tienen un tiempo de actividad médica que llega a 22 años, aproximadamente, y 5 años en la actividad de auditoría.

De igual forma, existe una relación significativa entre las variables "Años laborando" y "Niveles de competencias en auditoría". Este hallazgo es contrario al obtenido por Olivia Furiady ${ }^{(11)}$, que muestra en su investigación que la experiencia laboral no mejoraría la calidad de las auditorias contables, aunque aclara que tal resultado se debería a que en la población estudiada predominaron los auditores jóvenes.

Por otro lado, los niveles de competencias globales están poco desarrolladas en los participantes, lo cual es preocupante, ya que los directores de IPRESS son considerados como referentes críticos en la evaluación del comportamiento de un establecimiento (12). Estos hallazgos evidenciarían una brecha en conocimientos relacionados a temas administrativos, de gestión y control, y se explicarían porque el $59,40 \%$ de los participantes no siguió capacitaciones en auditoría, y el otro $50,00 \%$ no se capacitó en gerencia de servicios de salud.

Esta situación es similar a lo reportado por Libardo A. Girald (13) (Antioquía, Colombia), que determinó que los resultados globales del nivel de desarrollo de las competencias de los gerentes de las instituciones prestadoras de salud estaban, con frecuencia, en el nivel de novato $(41,00 \%)$ y básico $(38,00 \%)$, por lo que planteó que se diseñen estrategias para fortalecer dichas competencias.

Aunque las competencias evaluadas por Libardo A. Giraldo ${ }^{(13,14)}$ fueron exclusivas del área de gerenciamiento, existen similitudes y una relación estrecha con las competencias desarrolladas en auditoría, toda vez que la auditoría es un método poderoso utilizado durante la etapa de control del ciclo del gerenciamiento y mejoramiento de procesos. Asimismo, encontramos que las tres principales unidades funcionales que contribuyen a la calidad institucional son el desarrollo de las unidades de prestación de servicios, la planeación y control de la gestión ${ }^{(13)}$. Este hallazgo coincide con el plateamiento del "Informe Final de la Comisión Interventora del Seguro Integral de Salud 2017" (15).

De modo similar, se observó que estos valores difieren ligeramente de los determinados por Claus Michael Preger ${ }^{(10)}$ 
Niveles de competencias en auditoría de servicios de salud de los médicos del primer nivel de atención del distrito de Comas, 2018

en Rio Grande (Brasil), que muestran que el $67,60 \%$ de los participantes en su estudio tenían formación en auditoría, mientras que el $25,35 \%$ había seguido algún tipo de curso relacionado a gerencia en salud. Este hallazgo podría indicar que los profesionales extranjeros tienen mayor interés en formarse en áreas administrativas y control de la calidad; o tal vez sea debido a que las escalas salariales y la mayor oferta de cursos de esta naturaleza brindan mejores oportunidades de capacitación.

Cabe mencionar que se evidenció una relación significativa entre las variables "Formación general" (formación auditoria y gerencia) y "Niveles de competencia en auditoría"; tales resultados serían similares a los encontrados por Saravan (2), quien determinó que los programas educacionales en auditoría clínica deberían ser dictados a alumnos de posgrado porque aseguran el control de calidad en el cuidado del paciente y pueden brindar un valor agregado a los procesos de gestión del capital humano en la entidad (16).

Finalmente, de lo expuesto se puede mencionar que un gran porcentaje de los médicos del primer nivel de atención no han desarrollado competencias en auditoría de servicios de salud, situación que incrementaría el riesgo de brindar prestaciones sin cumplir los estándares de calidad en salud, así como el riesgo de dirigir de manera poco eficiente y eficaz las IPRESS del primer nivel de atención. Esta brecha propiciaría que los candidatos a directores de establecimiento de salud no cuenten con los conocimientos necesarios de gerenciamiento y control, por lo que estarían expuestos a incurrir en faltas por omisión o comisión durante el ejercicio de funciones públicas, hechos que podrían generar responsabilidades administrativas, civiles y hasta penales.

Por esto, es necesario que las Unidades de Gestión de las Instituciones Prestadoras de Salud (UGIPRESS) den mayor atención a dicha brecha e incluyan en los Planes de Desarrollo del Personal un mayor número de capacitaciones, cursos, programas y/o diplomados $(17,18)$ relacionados a estos temas, ya que las competencias son las que permiten un desempeño diferenciador y exitoso ${ }^{(19)}$ que brinde competitividad sostenible a la institución (20).

Contribuciones de los autores: Erick N. Guerra Uriarte se encargó del diseño de la investigación, recolección de datos, discusión de datos, análisis estadístico y análisis crítico.

Fuentes de financiamiento: Este artículo ha sido financiado por el autor.

Conflicto de interés: El autor declara no tener ningún conflicto de interés.

\section{REFERENCIAS BIBLIOGRÁFICAS}

1. Shah MN, Prakash A. Developing generic competencies for infrastructure managers in India. Int J Proj Manag. 2018.

2. Saravan T, Shanmugapriya S. A study on the effectiveness of teaching the principles and methods of clinical audit to medical postgraduates. Curr Med Res Pract. 2018; 8(2018): 59-63.

3. Llanos Zavalaga F. Auditoría en el primer nivel de atención. Rev Med Hered. 2000; 11(3): 107-12.

4. Ministerio de Salud. Documento Técnico: "Sistema de Gestión de la Calidad en Salud". RM n ${ }^{\circ}$ 519/2006/MINSA, de 30 de mayo [Internet]. Diario oficial El Peruano. Lima; 2006. Disponible en: http://bvs. minsa.gob.pe/local/dgsp/000_SGCALIDAD-1.pdf

5. Ministerio de Salud. Listado de Estándares de Acreditación para Establecimientos de Salud y Servicios Médicos de Apoyo de la Norma Técnica N ${ }^{\circ}$ 050-MINSA/DGSP-V02, Resolución Ministerial N ${ }^{\circ} 456.2007$ / MINSA, de 04 de junio [Internet]. Diario oficial El Peruano. Lima; 2007. Disponible en: http://www.minsa.gob.pe/comisiones/ acreditacion/normatividad.asp

6. Chirino-Muñoz MS. Competencias laborales del auditor de servicios de salud: un análisis funcional Venezuela, 2008. Rev Salud Pública. 2010; 12(5): 833-42.

7. Araya-Pizarro SC. Competencias genéricas de los estudiantes de Auditoría requeridas por las Big Four en Chile. Cuadernos de Contabilidad. 2019; 49(20).

8. Ministerio de Salud. Guía Técnica para la Identificación de Competencias y Diseño de Estándares e Instrumentos de Evaluación de Competencias Específicas con el Enfoque Funcional, Resolución Ministerial $n^{\circ} 251-2014 /$ MINSA, de 24 de marzo [Internet]. Diario ofical El Peruano. Lima; 2014. Disponible en: http://www.minsa. gob.pe/dggdrh/libros/pdf/s1/Minsa_oga_Guiatecnica.pdf

9. Ministerio de Salud. Guía Técnica del Evaluador para la Acreditación de Establecimientos de Salud y Servicios Médicos de apoyo, Resolución Ministerial n²70-2009/MINSA, de 23 de abril [Internet]. Diario oficial El Peruano. Lima; 2009. Disponible en: http://www. minsa.gob.pe/comisiones/acreditacion/normatividad.asp

10. Preger CM, Berger I, Gonçalves de Fonte CA, Mascarello HC. Perfil dos Médicos Auditores No Estado do Rio Grande do Sul. Rev Assoc Med Bras. 2005; 51(2): 87-92.

11. Furiady 0 , Kurnia R. The effect of work experience, competency, motivation, accountability and objectivity towards audit quality. Procedia Soc Behav Sci. 2015; 211: 328-35.

12. Rodríguez Córdoba MP, Bustamante Lozano U. Desarrollo de Competencias para el comportamiento ético-gerencial: un enfoque de responsabilidad. Cuad Adm Bogotá (Colombia). 2008; 21(35): 205-28.

13. Girlado LA, Grisales LM, Ortiz P. Relación entre el nivel de desarrollo de las competencias en los gerentes de las IPS y la calidad institucional, Antioquia, 2005. Rev Fac Nac Salud Pública. 2006; 26(2): 1-11.

14. Giraldo LA, Grisales LM. Identificación y normalización de las competencias en los gerentes de las instituciones prestadoras de servicios de salud. Rev Fac Nac Salud Pública. 2005; 23(2): 31-51.

15. Seguro Integral de Salud. Informe Final de la Comisión Interventora del Seguro Integral de Salud - Final [Internet]. Lima; 2017. Disponible en: https://cdn.www.gob.pe/uploads/document/ file/1086161/20170628_InformeFinalComisionInterventoraSIS.pdf

16. Gallego M. Gestión Basada en Competencias Contribución efectiva al logro de objetivos organizacionales. Revista universidad EAFIT. 2000; 36(119).

17. Chapman SJ, Glasbey JC, Khatri C, Michael K, Nepogodiev D, Bhangu $A$, et al. Promoting research and audit at medical school: evaluating the educational impact of participation in a student-led national collaborative study. BMC Medical Education. 2015; 47(15). 
18. STARSurg Collaborative. Medical research and audit skills training for undergraduates: An international analysis and student-focused needs assessment. Postgrad Med J. 2017.

19. Gérvas J. La Gerencia vista desde la Consulta, a través del prisma de la calidad asistencial. SEMERGEN. 2005; 31(1): 15-7.

20. Muñoz Arias J, Calderón Hernández G. Gerencia y competencias distintivas dinámicas en instituciones prestadoras de servicios de salud. Revista Gerenc Polit Salud. 2008; 7(15): 131-54.

\section{Correspondencia:}

Erick Nelson Guerra Uriarte

Dirección: Leonardo Arrieta 1420 Lima, Perú.

Teléfono: +51959587507

Correo electrónico: kriegerick@hotmail.com

\section{Recibido: 13 de noviembre 2020}

Evaluado: 04 de enero de 2021

Aprobado: 11 de febrero de 2021

( C La revista. Publicado por Universidad de San Martín de Porres, Perú.

(c) Br Licencia de Creative Commons Artículo en acceso abierto bajo términos de Licencia Creative Commons Atribución 4.0 Internacional. (http://creativecommons.org/licenses/by/4.0/)

ORCID iDs

Erick Nelson Guerra Uriarte 7 https: / / orcid.org/0000-0001-7640-0519 